\title{
Primary small intestinal epithelioid angiosarcoma: a potential diagnostic pitfall due to abnormal expression of cytokeratin
}

wen yuanyuan

Zhoushan Hospital

\section{Sangao Fang}

Third Military Medical University Daping Hospital and Research Institute of Surgery

Zhen Zheng

Shaoxing People's Hospital

Liyong Qian

Zhoushan Hospital

wei jianguo ( $\square$ mickmouse88@163.com )

\section{Case Report}

Keywords: small intestinal, jejunum, epithelioid angiosarcoma, immunohistochemistry

Posted Date: February 12th, 2020

DOI: https://doi.org/10.21203/rs.2.23381/v1

License: (c) (1) This work is licensed under a Creative Commons Attribution 4.0 International License.

Read Full License 


\section{Abstract}

Background: Primary small intestinal epithelioid angiosarcoma (EAS) is a very rare tumor, which originates from endothelial cell with high mortality. Only 66 cases of small intestinal angiosarcoma including primary or metastatic were reported in the current literature. Histologically, hemangiosarcoma with epithelioid cell morphology is more rare, so we report a case of EAS which is easily misdiagnosed as poorly differentiated carcinoma. We also reviewed the literature about small intestinal angiosarcoma.

Case presentation: The patient was a 60-year-old man, who initially presented black stool with no obvious inducement for 7 days, gradually feeling dizzy and fatigue for 3 days. Abdominal CT showed that the intestinal wall of the upper jejunum was thickened near the duodenum. After enhancement, the intestinal wall could be enhanced evenly. With the time going on, the contrast media could be concentrated gradually, and the active hemorrhage was considered. Enteroscopy revealed a $30 \mathrm{~mm} \times 20 \mathrm{~mm}$ red polypoid mass in the jejunum. A biopsy of polypoid mass exhibited malignant tumor, histopathology considered poorly differentiated cancer. The patient subsequently underwent resection of the jejunal neoplasm. Immunohistochemistry vascular markers and cytokeratin were postive区but C-MYC protein was

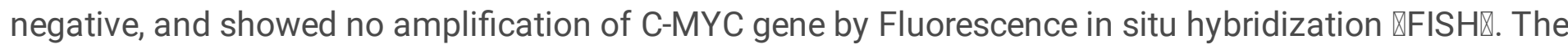
histopathology with immunohistochemical and FISH studies confirmed the diagnosis of primary small intestinal EAS. 24 months after surgery, the patient had no evidence of recurrence and metastasis.

Conclusion: Primary small intestinal EAS is a rare malignancy of endothelial origin with uncertain etiology and often has a poor prognosis.The abnormal cytokeratin expression in epithelioid angiosarcomas represents a potential diagnostic pitfall for pathologists. FISH detection of C-MYC gene amplification may have some value in the diagnosis of primary or metastatic small intestinal AS.An accurate pathological diagnosis relies on histopathology with immunohistochemical stains of endothelial markers. Surgical resection remains the best treatment option for small intestinal angiosarcoma.

\section{Introduction}

Angiosarcoma (AS) is a rare malignant sarcoma originating from vascular endothelial cells, accounting for less than $1 \%$ of all soft tissue sarcomas. Most frequently occur in head and neck skin and soft tissue[1, 2]. Small intestinal AS is very rare. To the best of our knowledge,only 66 cases of small intestinal angiosarcoma including primary or metastatic were reported in the current literature,most had only been found in scattered case reports and small series[3-8]. Pathologically AS can be classified into welldifferentiated, poorly differentiated,and epithelioid AS. In contrast to these common features,epithelioid angiosarcoma (EAS) is known as a unique morphologic subtype of AS, histopathologically the malignant endothelial cells have a predominantly epithelioid appearance. AS is highly invasive and often occur distant metastasis rapidly. Therefore, unless early finding and appropriate treatment are given, the prognosis is usually poor. At present, the etiology of AS is not clear[2-4]. Most pathologists lack knowledge of AS and are easily misdiagnosed as poorly differentiated adenocarcinoma, especially when 
tumor cells express epithelial markers abnormally in small biopsy specimens. The aim of this study is to raise awareness of this rare and highly aggressive neoplasm and differentiate it from poorly differentiated adenocarcinoma, epithelioid sarcoma, carcinosarcoma, and so on. In addition, there are few studies on C-MYC protein and gene amplification in gastrointestinal AS, so we preliminarily discuss the diagnostic value of C-MYC protein and gene amplification in gastrointestinal AS.

\section{Case Presentation}

\section{Clinical history}

A 60-year-old Chinese man, who initially presented black stool with no obvious inducement for 7 days, gradually feeled dizzy and fatigued for 3 days. The patient had poor spirit, appetite and sleep. There was no significant change in weight and no previous operation history. Blood routine test: red blood cell count $\left(2.40 \times 10^{12} / \mathrm{L}\right)$ and hemoglobin $(80 \mathrm{~g} / \mathrm{L})$ was decreased, fecal occult blood was positive.Abdominal CT showed that the intestinal wall of the upper jejunum was thickened near the duodenum. After enhancement, the intestinal wall could be enhanced evenly,with the time going on, the contrast media could be concentrated gradually(Figure 1), the fat space around the tumor was clear, and there was no swelling of the mesentery and retroperitoneal lymph nodes, and the active hemorrhage was considered. Gastroscopy showed a lesion in the horizontal segment of the descending duodenum, which was covered with a large number of dark red blood clots.Pathological biopsy showed chronic inflammation of mucosa. Enteroscopy revealed a $30 \mathrm{~mm} \times 20 \mathrm{~mm}$ red polypoid mass in the jejunum (Figure 2). He underwent jejunal tumor resection and jejunojejunostomy were performed. During the operation, it was found that below the duodenal suspensory ligament $5 \mathrm{~cm}$ touched the mass about $3 \mathrm{~cm} \times 3 \mathrm{~cm}$ in size, with medium texture and no obvious invasion and adhesion with surrounding organs. No radiotherapy and chemotherapy was given after operation, without recurrence or metastasis after 2 years follow-up.

\section{Pathological and immunophenotypic findings}

On gross examination, the excised jejunum intestine specimen measured $6.0 \mathrm{~cm}$ in length and $2.8 \mathrm{~cm}$ in pipe diameter, with a raised nodule measured $3.0 \mathrm{~cm} \times 2.0 \mathrm{~cm} \times 2.0 \mathrm{~cm}$ in size, the mass was located at $1.8 \mathrm{~cm}$ from the proximal margin. The mass mucosal surface was partially erosive; cross-sections of the tumor was dark brown with medium texture. Microscopically, hemorrhage and necrosis could be seen on the mucosal surface, involved the mucosa, submucosa(Figure 3A); blood lakes and hemosiderin deposits could be seen, the tumor cells were arranged in solid and flaky distribution. (Figure 3B), forming slitshaped,pseudoglandular or papillary structures(Figure 3C). A large number of red blood cells could be seen in the tumor, and the anastomotic vascular lining cells were spindle or epithelioid, the cytoplasm were eosinophilic, the nucleus were vacuolate, the nucleolus were prominent(Figure 3D), and the mitosis were easy to see. Blood vessels were connected and anastomosed(Figure 3E), and transition between well differentiated areas and poorly differentiated areas(Figure 3F). Immunohistochemically, neoplastic cells were postive for CD34(Figure 4A),CD31(Figure 4B),ERG(Figure 4C),Fil-1, factor VIII,INI1; Partial tumor cells were positive for cytokeratin(CK) (Figure 4D); but were negative for 
CK7,CK20,EMA,CD117,D0G1,S100,SMA,CD68,D2-40,C-MYC protein,HHV8.Fluorescence in situ hybridization(FISH) did not show C-MYC gene amplification. The final histological diagnosis was primary small intestinal epithelioid angiosarcoma.

\section{Discussion}

AS is a highly malignant soft tissue sarcoma originated from vascular endothelial cells, accounting for less than $1 \%$ of soft tissue sarcoma[1]. It often occurs in the skin, subcutaneous tissue of the head and face[9],breast, but also in the mouth[10], mediastinum[11], kidney[12], thyroid[13], lung and other anatomical sites[14]. AS in digestive system is rare, but in solid organs such as liver and spleen is relatively common[15]. Small intestine AS is particularly rare, to the best of our knowledge, only 66 cases of small intestinal angiosarcoma including primary or metastatic were reported in the current English literature. The most common sites of small intestinal AS were ileum and jejunum[2-8]. Our case was located in jejunum.

The etiology of AS remains unclear.It may be associated with long-term exposure to thorium oxide, vinyl chloride, arsenic and other chemicals. Radiation export may also be the cause of AS,such as radiotherapy treatment for prostate cancer, colorectal cancer, endometrial cancer or cervical cancer[2-4].

Chemotherapy,chronic lymphedema, chronic inflammation,and trauma may also be associated with angiosarcoma. It had been reported that angiosarcoma was probably associated with long-term taking of calcium channel blocker to control blood pressure,such as levamlodipine besylate[2]. It may occur around arteriovenous fistula or persistent foreign body. In addition, there is a certain relationship with hemodialysis or genetic factors. The average time from exposure to these factors to the diagnosis of angiosarcoma was 18 years (range of 4 to 30 years) [3]. Our patient did not have any of these aforementioned medical histories.

Li et al. reviewed the literature found 66 cases of small intestinal AS[4]. There were 24 patients diagnosed before the year 2000, and 42 patients were diagnosed after 2000. Patient age ranged from 24 to 92 years, with an average age was 64 year old, and $70 \%$ of patients were over 60 years old. The female:Male ratio was 1:2.4. The clinical manifestations of small intestine AS were extremely variable and nonspecific. The main clinical features included gastrointestinal bleeding (black stool, hematochezia or occult bleeding), abdominal discomfort/pain, and abdominal mass. Other symptoms included intestinal obstruction, diarrhea and weight loss, nausea/vomiting, acute abdomen,bowel perforation,skin pallor/ anemia, etc[28]. Some early cases were even asymptomatic and were occasionally found in routine endoscopy[16]. Our patient developed black stool for 7 days without obvious inducement, and gradually felt dizzy and weak for 3 days.Because the clinical symptoms are nonspecific,and may be confused with other gastrointestinal diseases, it is easy to misdiagnose. To some extent biopsy is helpful to definite diagnosis, but postoperative pathological examination is still the most effective way to definite diagnosis. 
Endoscopy reveals that AS is generally nodular, polypoid or protuberant mass, most are solid, a few may be spongy, diffuse or ulcerative. Cross-sections of the tumor are usually soft, accompanied by hemorrhage, necrosis and fish-like change. Pathological examination tumor diameter was $1.5 \mathrm{~cm} \sim$ $12.0 \mathrm{~cm}$, with an average diameter of $5.0 \mathrm{~cm}$. It is usually a single nodule, occasionally a multiple nodule[6].Histologically, AS can be classified into well-differentiated, poorly differentiated,and epithelioid tumors. 42 reports provided detail pathological description, of which 17 cases were epithelioid, 10 cases were well differentiated, 11 cases were poorly differentiated[4].Microscopically, the tumor cells are spindle, epithelioid or irregular in shape, with marked heteromorphism, common mitosis,necrosis, prominent nucleoli and rich eosinophilic cytoplasm,forming slit-shaped or pseudoglandular or papillary structures. The most significant diagnostic features are irregular vascular anastomosis and atypical endothelial cells, with red blood cells in the lacuna. In addition, the formation of blood lake is of great significance for diagnosis.

Immunohistochemistry is the better way to make an accurate diagnosis of the epithelioid AS. AS immunohistochemistry expresses more than one vascular endothelial markers, including: CD34, CD31, ERG, FLI-1,factor VIII, UEA-1 and so on[17]. The factor VIII-associated antigen and UEA-1 are considered to be the most sensitive and reliable markers in evaluating the origin of epithelioid cell. Factor VIII has a high specificity for the diagnosis of AS, but is not very sensitive to poorly differentiated AS. UEA-1 has high sensitivity but low specificity[18].The expression rate of CD34 in gastrointestinal tract AS is significantly higher than that in skin AS. In the diagnosis of AS, the sensitivity of CD31 is better than the factor VIII, and its specificity is higher than UEA-1[18]. Usually one or more vascular markers are positive, and AS is diagnosed when the epithelial markers are negative. Although some vascular endothelial cell markers can also stain histiocytes, megakaryocytes, myeloid cells, or some other types of cells, our experience is that more than two strong and diffusely stained endothelial markers appear simultaneously in malignant solid tumors. At this time, the diagnosis of AS has high specificity (except epithelioid sarcoma and myeloid sarcoma). Recently, the ETS family transcription factors ERG and FLI-1 have been considered as relatively specific markers of vascular endothelium[19]. Without these markers, AS is easily misdiagnosed as differential cancer. It should be pointed out that the expression rate of ERG in well-differentiated AS is higher, while in poorly-differentiated or epithelioid AS, the expression may be lost due to the dedifferentiation of the tumor. However, epithelioid AS can focally express epithelial markers (CK7 and CK), and can also express CK when AS metastasizes to lymph nodes, which may bring some pitfalls in biopsy. In addition, CD117 is not routinely used as a vascular-derived marker, but when AS is positive, it suggests that patients receiving imatinib treatment may be effective[20].

Some results showed that the amplification of C-MYC gene on chromosome 8q24.21 had high sensitivity and specificity for secondary AS (including radiation-related AS and lymphedema related AS), and there was overexpression of C-MYC protein simultaneously, while the amplification of C-MYC gene was rare in primary cutaneous AS[21]. The overexpression of C-MYC protein in immunohistochemistry is not specific to primary or secondary AS. Different mechanisms (any link including gene amplification, gene rearrangement, transcription, translation or post-translational modification) may lead to the overexpression of C-MYC protein[22]. Therefore, we can not differentiate primary or secondary AS by the 
overexpression of C-MYC protein by immunohistochemistry alone, we must detect whether there is amplification of C-MYC gene by FISH. Our patient had no medical history of AS or the other tumor in other anatomical site. Immunohistochemistry C-MYC protein was negative, and showed no amplification of CMYC gene by FISH, all of the above results supported primary small intestine AS.

Before diagnosing primary small intestinal AS, it is necessary to exclude metastatic AS firstly. The clinical history is very important. The differential diagnosis mainly includes (1) gastrointestinal stromal tumor (GIST): The simultaneous expression of CD117 and DOG1 has higher sensitivity and specificity for GIST. CD34 is also positive in GIST, but its specificity is relatively low. (2) Poorly differentiated cancer: tumor cell nuclear polymorphism is more obvious, and vascular fissure-like structures and blood lake phenomena are rarely seen. Immunohistochemistry expressed CK but did not express vascular markers. (3) Epithelioid sarcoma: sometimes it also can produce pseudohemangioma-like morphology, which can express Vimentin, CK, CD34,it has immunophenotype overlap with epithelioid AS, but usually INI-1 is negative, and CD31 and ERG are not expressed. (4) Kaposi's sarcoma(KS): It is usually associated with immunosuppression such as AIDS. The small intestinal KS is usually multifocal. The fissure-like space contains red blood cells between the spindle cells, PAS positive eosinophilic transparent bodies can be seen, it can also express vascular endothelial markers, but HHV8 positive can help diagnosis[23].(5) Malignant mesothelioma: It expresses mesothelial markers such as CK5/6, WT-1, D2-40, Calretinin,etc, but does not express vascular endothelial markers. (6) Carcinosarcoma: It's composed of cancer and sarcoma, the component of sarcoma can be for AS. Cancer and sarcoma have different immunophenotypes[24].

The factors influencing the poor prognosis include age, tumor size, TNM stage, surgical margin, and metastasis[3]. The patients' age is less than 50 years old, tumor diameter is less than $5.0 \mathrm{~cm}$, low TNM stage and negative surgical margin are all relatively good prognostic factors. The small intestinal AS commonly metastasizes to regional lymph nodes,the liver, lungs, and bones. Metastases at presentation occur in $40 \%$ of the cases[3, 4].

Because small intestinal AS is rare, there is no standard treatment guidelines. Usually surgery or postoperative radiotherapy and chemotherapy is the treatment option. Surgical resection is the first choice for focal resectable tumors, which may also be used for palliative treatment in patients with metastatic disease. For localized AS, complete radical resection with wide margins is the primary treatment of choice[3,4]. Early recognition of the clinical signs and symptoms with early diagnosis and complete surgical resection should be beneficial. Whether neoadjuvant therapy is useful for advanced diseases remains controversial. Prognosis in advanced diseases is very poor despite the use of surgery, chemotherapy and even radiotherapy. Surprisingly, there is a case of spontaneous regression of pulmonary metastatic EAS from the small intestine[5].Because of the limited scope of tumor involvement and negative surgical margin, our patients had relatively good prognosis. No radiotherapy and chemotherapy was given after operation, without recurrence or metastasis after 2 years follow-up.

\section{Conclusions}


Small intestinal AS is a rare but aggressive malignancy of endothelial origin. A definitive pathological diagnosis relies on histopathology with immunohistochemical stains of endothelial markers. Immunohistochemical expression of epithelial markers may be misdiagnosed as poorly differentiated adenocarcinoma. FISH detection of C-MYC gene amplification may have some value in the diagnosis of primary or metastatic small intestinal AS.Surgical resection is still the best choice of the different treatment options.

\section{Declarations}

\section{Acknowledgements}

None.

\section{Authors' contributions}

Both authors performed the histological and immunohistochemical evaluation as well as collected clinical data and drafted the manuscript. Both authors read and approved the final manuscript.

\section{Funding}

Project of natural science foundation of zhejiang province LY16H160058;Medical and health science and technology project of zhejiang province 201512180;Shaoxing public welfare Technology Application Research Project 2017B70020.

\section{Availability of data and materials}

The datasets during and/or analysed during the current study available from the corresponding author on reasonable request.

\section{Authors' contributions}

WYY and FSG performed pathologic diagnosis and writing of manuscript and collected clinical data and follow-up of the patient.ZZ and QLY contributed in searching literature, performed immunohistochemical. WJG contributed to the pathologic diagnosis and revised the manuscript. All authors read and approved the final manuscript prior to submission.

\section{Ethics approval and consent to participate}

Not applicable.

\section{Consent for publication}

Written informed consents for publication of their clinical details and/or clinical images were obtained from the patient. A copy of the consent forms is available for review by the Editor of this journal. 


\section{Competing interests}

The authors declare that they have no competing interests.

\section{Author details}

${ }^{1}$ Department of Pathology, Zhoushan Hospital, Zhoushan, Zhejiang, 316000,China.

Email:wenyuanyuan1022@sina.cn

${ }^{2}$ Department of Pathology,Daping Hospital and Research Institute of Surgery,Army Medical University,Chongqing 400042,China.Email: fangsangaode@163.com

${ }^{3}$ Department of Pathology, Shaoxing People's Hospital, Shaoxing, Zhejiang, 312000, China. Email: mickmouse88@163.com

*Correspondence: mickmouse88@163.com

${ }^{\dagger}$ Yuanyuan Wen and Sagano Fang contributed equally to this work.

\section{Abbreviations}

AS: angiosarcoma; EAS:epithelioid angiosarcoma;GIST: gastrointestinal stromal tumor; ETS:E-twenty-six; FLI-1:Freund leukemia integration site1;CK:cytokeratin;CK7:cytokeratin 7; CK20:cytokeratin 20; CK5/6: cytokeratin5/6;EMA:epithelial cell membrane antigen;SMA: smooth muscle actin;

\section{References}

1. Cao J, Wang J, He C, Fang M.Angiosarcoma: a review of diagnosis and current treatment. Am J Cancer Res.2019;9(11):2303-2313.

2. Wang Q, Zhao T, Mi BT, Zhang YL, Wei R, Tong HL, et al.Primary Colonic Angiosarcoma Seen in a Patient on Calcium Channel Blocker: A Case Report with Summary Analysis of 32 Other Cases from the Literature. Am J Case Rep.2018,19(2):254-261.

3. Nai Q, Ansari M, Liu J, Razjouyan H, Pak S, Tian Y, et al.Primary Small Intestinal Angiosarcoma: Epidemiology, Diagnosis and Treatment. J Clin Med Res.2018;10(4):294301.

4. Li R, Ouyang ZY, Xiao JB, He J, Zhou YW, Zhang GY, et al.Clinical Characteristics and Prognostic Factors of Small Intestine Angiosarcoma: a Retrospective Clinical Analysis of 66 Cases. Cell Physiol Biochem.2017;44(2):817-827.

5. Hori S, Tachihara M, Tamura D, Kobayashi K, Nakata K, Kamiryo H, et al.Spontaneous Regression of Epithelioid Angiosarcoma in a Young Woman. Intern Med.2017;56(24):3333-3339.

6. Navarro-Chagoya D, Figueroa-Ruiz M, López-Gómez J, Nava-Leyva H, Álvarez-Ponce CE, GuzmánSombrero $\mathrm{G}$, et al.Obscure gastrointestinal bleeding due to multifocal intestinal angiosarcoma. Int J 
Surg Case Rep.2015;10:169-72.

7. Ni Q, Shang D, Peng H, Roy M, Liang G, Bi W, et al.Primary angiosarcoma of the small intestine with metastasis to the liver: a case report and review of the literature. World J Surg Oncol.2013;11:242.

8. Zacarias Föhrding L, Macher A, Braunstein S, Knoefel WT, Topp SA.Small intestine bleeding due to multifocal angiosarcoma. World J Gastroenterol.2012;18(44):6494-500.

9. Shustef E, Kazlouskaya V, Prieto VG, Ivan D, Aung PP.Cutaneous angiosarcoma: a current update. J Clin Pathol.2017 Nov;70(11):917-925.

10. Nagata M, Yoshitake Y, Nakayama H, Yoshida R, Kawahara K, Nakagawa Y, et al. Angiosarcoma of the oral cavity: a clinicopathological study and a review of the literature. Int J Oral Maxillofac Surg.2014;43(8):917-23.

11. Tan YB, Yu XF, Fan JQ, Li JF.Angiosarcoma originating in the anterior mediastinum: A case report. Medicine (Baltimore).2018;97(50):e13459.

12. Mastoraki A, Schizas D, Giannakas T, Papadopoulos PP, Naar L, Vergadis C, et al.Primary Angiosarcoma of the Kidney: Literature Review of a Rare Nosologic Entity. Anticancer Res.2020;40(2):625-633.

13. De Felice F, Moscatelli E, Orelli S, Bulzonetti N, Musio D, Tombolini V.Primary thyroid angiosarcoma: A systematic review. Oral Oncol.2018;82:48-52.

14. Faiek S, Tariq H, Upparapalli D, Bansal A, Sompalli S. Primary Pulmonary Epithelioid Angiosarcoma: A Rare Case Presentation of Bilateral Pneumothoraces. 2019;11(12):e6514.

15. Yasir S, Torbenson MS.Angiosarcoma of the Liver: Clinicopathologic Features and Morphologic Patterns. Am J Surg Pathol.2019;43(5):581-590.

16. Saad A, Cappell MS, Amin M.Endoscopic findings with GI angiosarcoma correspond with the propensity of these vascular tumors to cause GI bleeding: two case reports and review of the literature. Dig Dis Sci. 2013;58(6):1797-801.

17. Xia J, Shi D, Wu Z, Chen Y, Liu B, Chen L, et al.The poor prognosis of the primary gastric epithelioid angiosarcoma: A case report. Medicine (Baltimore).2018;97(15):e0287.

18. Orchard GE, Zelger B, Jones EW, Jones RR.An immunocytochemical assessment of 19 cases of cutaneous angiosarcoma. 1996;28(3):235-40.

19. Stockman DL, Hornick JL, Deavers MT, Lev DC, Lazar AJ, Wang WL.ERG and FLI1 protein expression in epithelioid sarcoma. Mod Pathol.2014;27(4):496-501.

20. Liu L, Kakiuchi-Kiyota S, Arnold LL, Johansson SL, Wert D, Cohen SM.Pathogenesis of human hemangiosarcomas and hemangiomas. Hum Pathol.2013;44(10):2302-11.

21. Shon W, Sukov WR, Jenkins SM, Folpe

AL.MYC amplification and overexpression in primary cutaneous angiosarcoma: a fluorescence insitu hybridization and immunohistochemical study. Mod Pathol.2014;27(4):509-15.

22. Laé M, Lebel A, Hamel-Viard F, Asselain B, Trassard M, Sastre X, et al.Can Cmyc amplification reliably discriminate postradiation from primary angiosarcoma of the breast? 
Cancer Radiother.2015;19(3):168-74.

23. Halankar J, Martinovic E, Hamilton P.Kaposi's Sarcoma Presenting as Acute Small Bowel Obstruction Diagnosed on Multidetector Computed Tomography with Histopathological Correlation. Case Rep Radiol.2015;2015:581470.

24. Cuadra-Urteaga JL, Font A, Tapia G, Areal J, Taron M.Carcinosarcoma of the upper urinary tract with an aggressive angiosarcoma component. Cancer Biol Ther.2016;17(3):233-6.

\section{Figures}
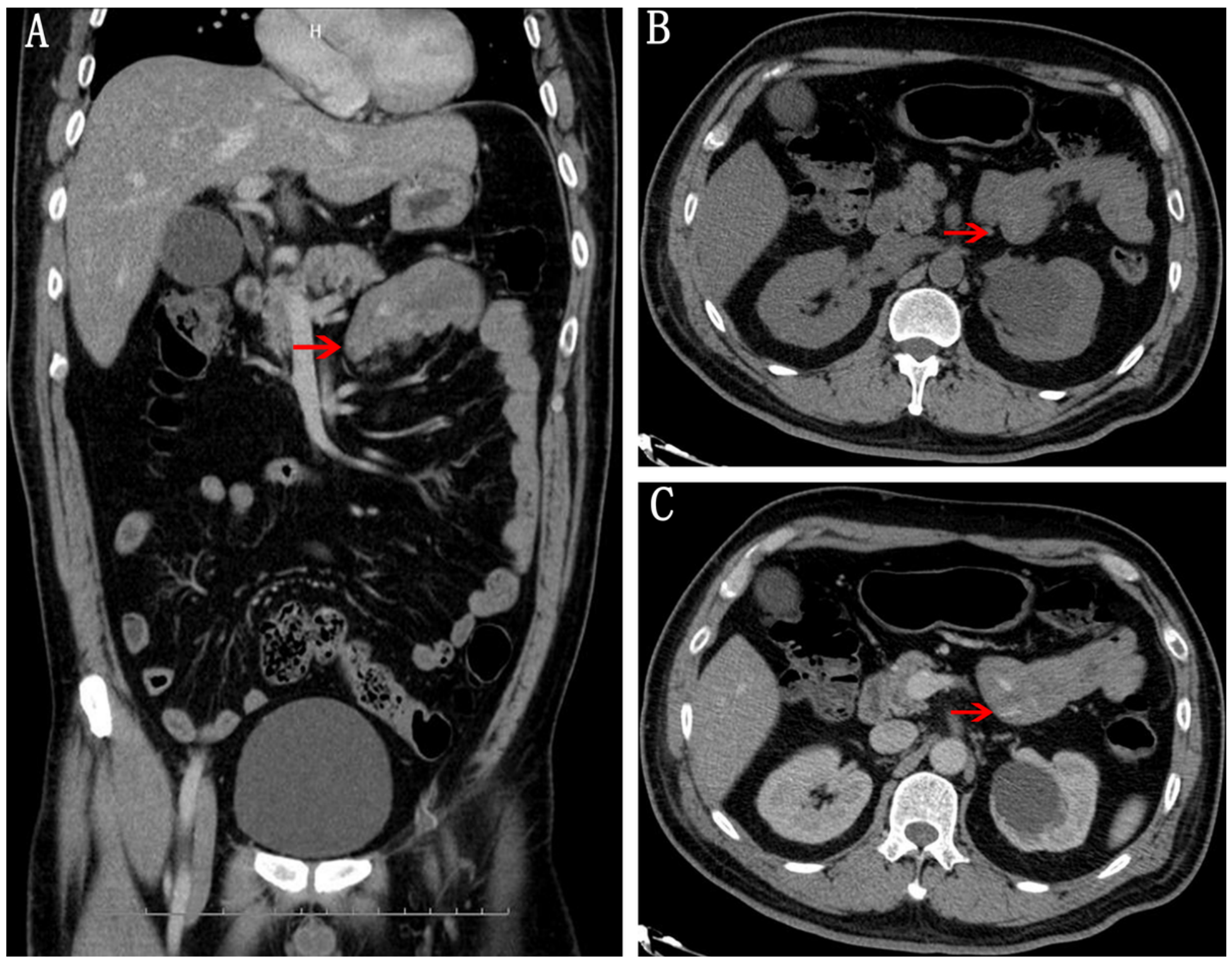

Figure 1

Abdominal CT showed that the intestinal wall of the upper jejunum was thickened near the duodenum. After enhancement, the intestinal wall could be enhanced evenly(A $B$ and $C$ ). 


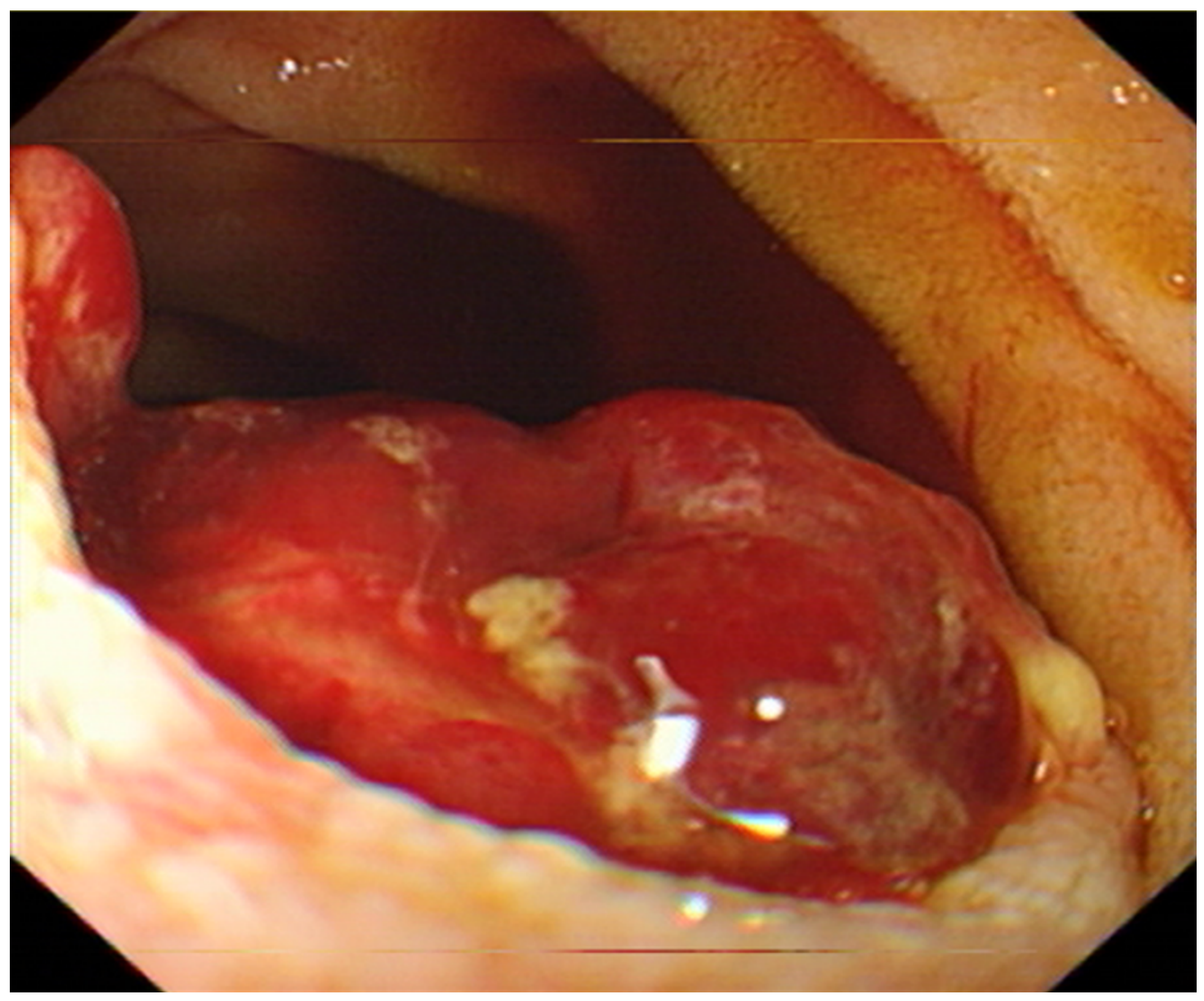

Figure 2

Enteroscopy revealed a $30 \mathrm{~mm} \times 20 \mathrm{~mm}$ red polypoid mass in the jejunum.

展

Figure 3

Microscopic features of hematoxylin-eosin stained paraffin sections of EAS. (A) Hemorrhage and necrosis could be seen on the mucosal surface, involved the mucosa, submucosa. $\otimes \mathrm{B} \otimes T$ The tumor cells were arranged in solid and flaky distribution囚and the formation of blood lakes.(C) Forming slitshaped,pseudoglandular or papillary structures.(D) A large number of red blood cells could be seen in the tumor, and the anastomotic vascular lining cells were spindle or epithelioid, the cytoplasm were eosinophilic, the nucleus were vacuolate, the nucleolus were prominent.(E) Blood vessels were connected 
and anastomosed,and forming blood lakes.(F) Transition between well differentiated areas and poorly differentiated areas.
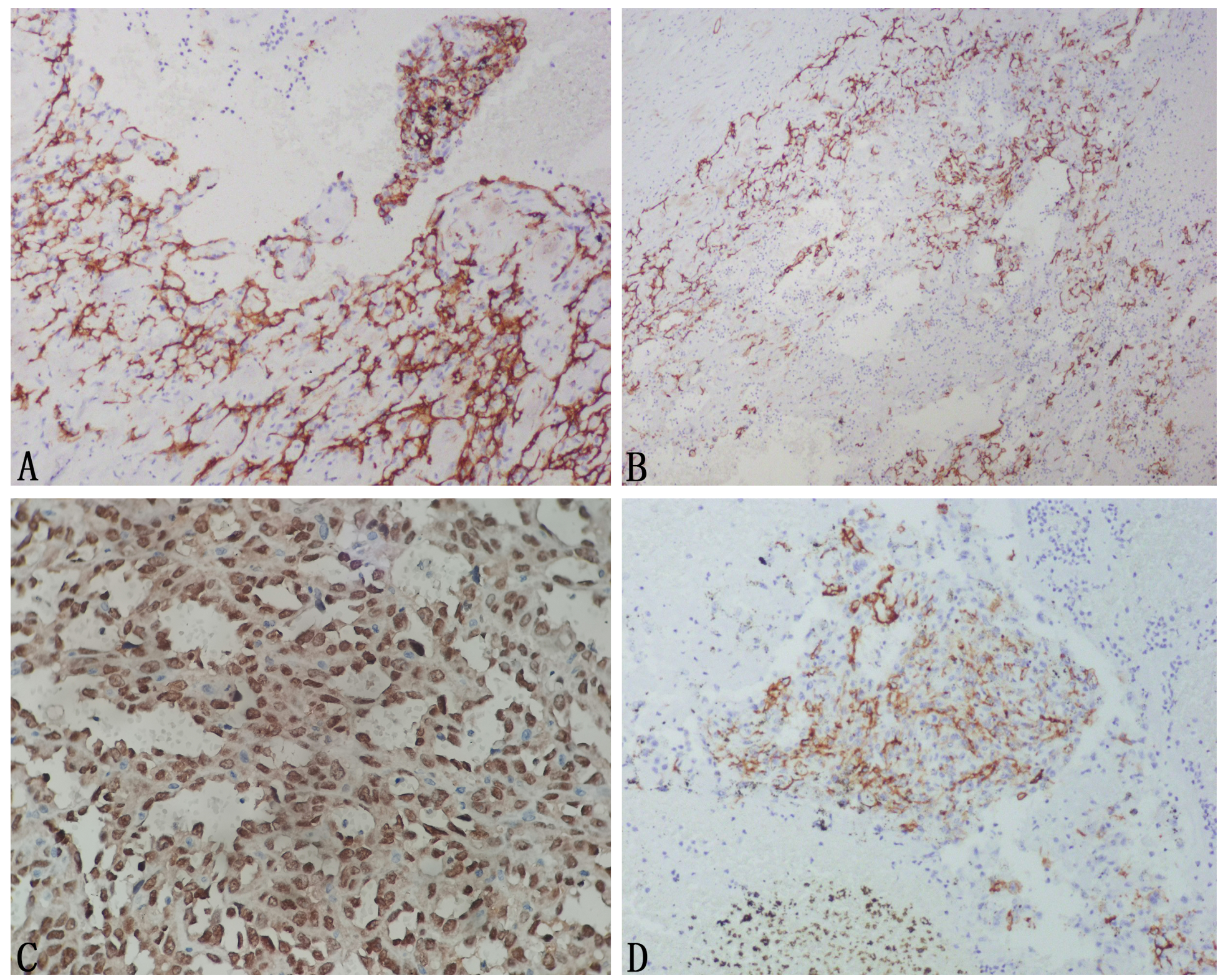

Figure 4

Positive immunostaining of EAS for vascular endothelial markers(A: CD34, B: CD31,C:ERG) and epithelial markers(D:CK)

\section{Supplementary Files}

This is a list of supplementary files associated with this preprint. Click to download.

- renamedf232c.jpg 